\title{
Relative motion: Kinetic information for the order of depth at an edge
}

\author{
ALBERT YONAS, LINCOLN G. CRATON, and WILLIAM B. THOMPSON \\ University of Minnesota, Minneapolis, Minnesota
}

\begin{abstract}
A new source of kinetic information for depth at an edge was investigated with adult subjects. The relationship between the motion of optical texture, indicating a surface, and the motion of a contour, indicating an edge, determines whether the surface is perceived as occluding or occluded. Subjects viewed computer-generated random-dot displays in which this relative-motion information provided the only information for depth order and a second type of display in which order in depth was specified both by relative-motion information and by the accretion and deletion of texture. Reliable depth effects were obtained in both conditions. These results indicate that adults are sensitive to the relative motion of texture and contour as information for depth at an edge.
\end{abstract}

Some depth cues (e.g., binocular disparity) provide the visual system with metric information for spatial layout, specifying the amount of depth separating two objects, whereas other cues (e.g., static interposition) provide only ordinal information. The latter type of cue indicates that a surface is either in front of or behind another, without providing information about the amount of separation in depth. Depth cues may also be classified as static or kinetic. Until recently, most theories of spatial perception have emphasized static depth cues and have paid little attention to the information carried by motion. Gibson (1950) challenged this approach when he pointed out that optical motions resulting from motion of the observer and/or objects provide a rich source of information for detecting the spatial layout of the environment. The purpose of this paper is to describe a potential source of kinetic information for the order of surfaces in depth and to demonstrate that humans are sensitive to this information.

Michotte, Thines, and Crabbe (1964) described a type of display in which purely kinetic information generated the perception of order in depth of two surfaces. Michotte et al. projected a series of forms on a screen, the first of which was a complete circular disk against a dark background. As the sequence continued, more and more of the circle was "blacked out," until it eventually disappeared (see Figure 1). This sequence was also presented in the reverse order. In both cases, the rectilinear portion of the edge of the disk underwent lateral motion while the curved portion appeared immobile. Subjects who

\footnotetext{
This work was supported by NICHD Grant HD-16924 and by Air Force Office of Scientific Research Contract AFOSR-86-0007. The authors wish to thank Herber Pick for the suggestion that we use a subjective contour display, Kim Pearson and Chi Ping Sze for programming the displays, Martha Arterberry and Herbert Pick for comments on an earlier draft of this manuscript, and Kaye $O$ 'Geay for typing the manuscript. Correspondence and requests for reprints should be sent to Albert Yonas, Institute of Child Development, University of Minnesota, 51 East River Road, Minneapolis, MN 55455.
}
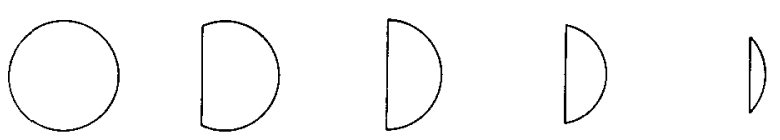

Figure 1. Schematic drawing of successive frames from the screening effect display created by Michotte, Thines, and Crabbe (1964).

viewed these displays reported seeing an unchanging circular disk being covered and uncovered by a second surface. Michotte et al. (1964) referred to this and similar phenomena as the screening effect. They maintained that Gestalt laws of perceptual organization accounted for the effect by completing and giving phenomenal permanence to the transforming disk.

Both Gibson's $(1966,1979)$ notion of an ecological optics and Marr's (1982) notion of a computational level for understanding vision argue for an analysis of the structure of the real world and the patterns of proximal stimulation that result from this structure that would make perception of spatial layout possible. Gibson (1966) described the stimulus information in Michotte's displays of the screening effect as the progressive "wiping out" or "unwiping" of optical texture that, he maintained, occurs whenever one surface is covered or uncovered by a second surface. Gibson and his students (Gibson, Kaplan, Reynolds, \& Wheeler, 1969) provided a second description of the change in the structure of the optic array that occurs when a surface is covered and uncovered by another:

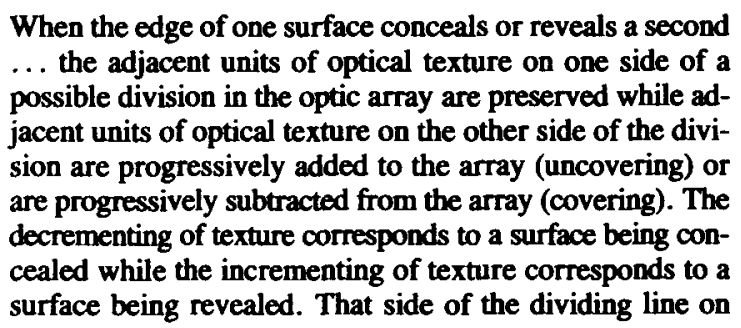


which there is deletion or accretion always corresponds to the surface that is behind; that side on which there is neither, always corresponds to the surface that is in front. (p. 114)

(It should be noted that the above analysis is not in fact correct for the rotation in depth of smooth surfaces. In such situations, the visible surface of the object is occluding itself. The side of the object boundary on which deletion or accretion is occurring is actually in front of the surface on the other side of the boundary.)

Kaplan (1969) tested two versions of the accretion/deletion hypothesis by presenting adult subjects with an animated film of random texture undergoing progressive accretion and deletion. When these displays are stationary, the viewer perceives a single textured surface. When the texture in these displays undergoes lateral motion, a vertical "subjective contour" is perceived at the vertical margin where texture elements are accreted and deleted. Kaplan presented subjects with three types of accretion/deletion displays. In the first type, texture was accreted or deleted on one side of a stationary vertical subjective contour while texture on the other side of the contour was preserved. This condition tested Gibson's hypothesis that whenever there is accretion/deletion of units of optical texture on one side of the contour and preservation of optical texture on the other side, a depth edge is perceived such that the region undergoing accretion/deletion is seen as a surface that is occluded. The second type of display used by Kaplan tested his own hypothesis that it is the region that undergoes the greater amount of accretion/deletion per interval of time that is perceived as a surface that is occluded. In this condition, texture was accreted or deleted simultaneously on both sides of the subjective contour, at varying relative rates. In a third type of display used by Kaplan, texture was simultaneously accreted or deleted at varying relative rates on both sides of a laterally moving subjective contour. This last condition was critical in that it allowed Kaplan to keep the rate of accretion/deletion constant while varying the velocity and direction of motion of the two surfaces defined by the contour in the display. Kaplan found support for the more general hypothesis that given a difference in the rate of accretion/deletion on the two sides of the subjective contour, adults will perceive depth at an edge.

However, both Kaplan's displays and those used in more recent studies of kinetic occlusion with adults (Andersen \& Braunstein, 1983) and infants (Granrud et al., 1984) contain another potential source of information for the covering and uncovering of one surface by another. Thompson, Mutch, and Berzins (1985) have observed that the order of surfaces in depth is specified by the relationship between the optical motion of a contour and the optical motion of the texture elements on either side of the contour. The principle underlying this account is that, for translational motion, the image of an occluding edge moves with the image of the occluding surface to which it belongs. Figure 2 illustrates the effect for simple translational motion. Shown in the figure are the optical mo- tion of texture elements corresponding to two surfaces and the optical motion of a contour corresponding to a depth edge. In Figure 2a, the left surface is in front and occluding the surface to the right. In Figure $2 b$, the left surface is now behind and being occluded by the surface to the right. The two cases can be distinguished because in Figure 2a the contour moves to the left, whereas in Figure $2 b$ the contour moves to the right.

Although the preceding analysis indicates that relative motion of contour and surface texture is physically determined by the order of surfaces in depth, it is not known whether this information is picked up by the human visual system. In the present study, therefore, we attempted to answer the question: does the relative motion of texture and contour determine the perception of depth at an edge? We investigated this question by presenting subjects with kinetic random-dot displays in which texture on both sides of a centrally located vertical contour was separated from the contour by blank space. This "gap" allowed for lateral motion of texture and contour without providing the subject with accretion/deletion information for depth at an edge. We hypothesized that when a contour and texture on one side of the contour moved together, subjects would perceive a continuous surface that was closer in depth than the surface defined by texture on the other side of the contour, whose motion was not tied to the motion of the contour. The size of the gap between texture and contour was varied to explore whether the detection of relative motion information for depth order was influenced by the spatial separation of the motions. A mechanism that detected relative-motion information might, we thought, function locally and involve processes with relatively small receptive fields. If this were the case, the perception of depth order would become ambiguous as the width of the gap between texture and contour was increased. If the mechanism were more global and integrated information over a large area, the size of the gap should have no effect on the perception of the order of surfaces in depth.

We also investigated whether or not visual mechanisms that detect information for the order of surfaces in depth were able to utilize different sorts of contour information equally effectively. Stimulus displays thus included both objective-contour conditions, in which an ordinary vertical line served as a contour, and subjective-contour conditions. Subjective contours are edges perceived in static displays in the absence of luminance differences (Kanizsa, 1955, 1979; Schumann, 1904). As previously mentioned, subjective contours also occur in conjunction with the depth effect produced by Kaplan's (1969) accretion/deletion displays. We hypothesized that both objectivecontour and subjective-contour conditions would produce reliable depth effects.

\section{METHOD}

\section{Subjects}

Sixteen unpaid students at the University of Minnesota, 15 undergraduates and 1 graduate student, served as subjects. 


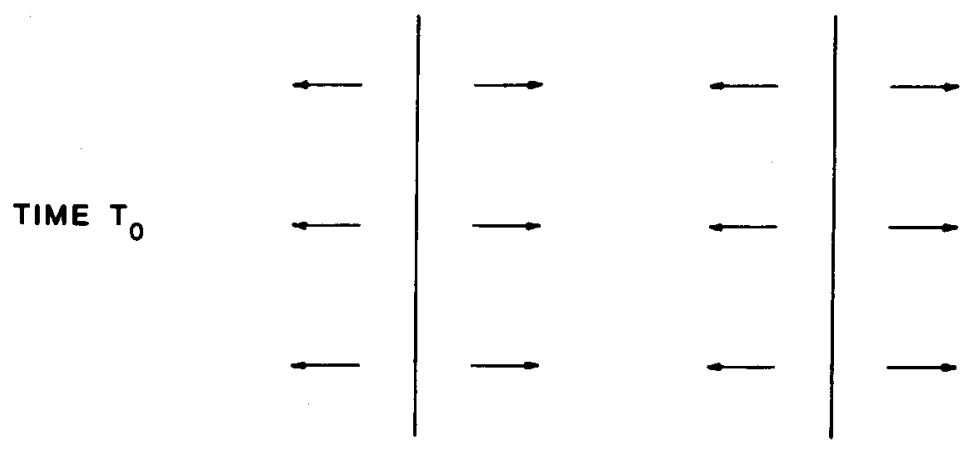

(a)

(b)

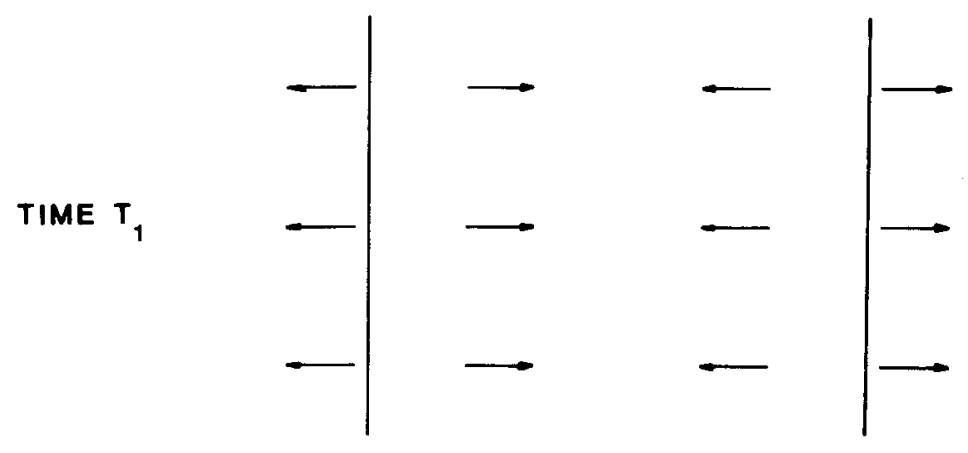

Figure 2. Motion of texture, indicated by arrows, and contours, indicated by change in position of vertical line from $T_{0}$ to $T_{1}$, specifies in (a) that the surface to the left of the line occludes the surface to the right. In (b), the left surface is occluded by the right surface.

\begin{abstract}
Apparatus
A TERAK microcomputer was used to generate random-dot displays containing kinetic information for the order of surfaces in depth. Displays were presented on a CRT and observed through an 11-cm-high and 21-cm-wide aperture (viewing distance, $.91 \mathrm{~m}$; visual angle, $6.9^{\circ}$ vertically and $13^{\circ}$ horizontally) attached to the face of the CRT. An eyepatch worn over one eye eliminated binocular information. A small box with three buttons labeled "left," "equal," and "right," respectively, was located directly in front of the subject. A 71/2-W nightlight provided dim overall illumination for the experimental room, sufficient for the dark-adapted subjects to see the response buttons.
\end{abstract}

\section{Displays}

Eight display conditions were presented. In each of these conditions, a vertical contour was displayed in the center of the screen. Randomly distributed texture elements to the left and right of the contour formed two texture fields. The texture fields contained approximately 2 dots per square centimeter. All the texture elements in a texture field moved in synchronous horizontal motion across the screen at $2 \mathrm{~cm} / \mathrm{sec}$. Texture on the left and right traveled simultaneously in opposite directions for a distance of $1 \mathrm{~cm}$ and then reversed direction. Thus, the two texture fields alternately approached and receded from one another. This pattern was repeated continuously. On each trial, the lateral motion of the vertical contour in the center of the screen was identical to that of one of the two texture fields. The eight display conditions were as follows:
Display Condition 1. Displays in the accretion/deletion (subjective contour) condition (see Figure 3a) were a modified version of those used by Kaplan (1969). Lateral movements of a subjective contour were accompanied by the appearance and disappearance of individual texture elements, resulting in the progressive accretion and deletion of texture elements on the left side of the display. In addition, the lateral motion of the texture field on the right side of the display was identical to the motion of the contour, whereas the motion of the texture field on the left side was not tied to that of the contour. Thus, in this condition, information for depth was provided by both the accretion and deletion of texture elements and the relative motion of texture and the contour.

Display Condition 2. Displays in the accretion/deletion (objectivecontour) condition were identical to those in the accretion/deletion (subjective-contour) condition, except that the central contour was a vertical line rather than a subjective contour. Again, the relative motion of both texture and contour and the accretion and deletion of texture elements provided information for depth.

Display Conditions 3, 4, and 5 . In the relative-motion (objectivecontour) condition, the relative motion of texture fields and the contour provided the only information for depth (see Figure 3b). Because of the presence of a textureless "gap" between texture fields and contour, this type of display eliminated accretion/deletion information while preserving relative-motion information. Texture on one side (in Figure 3b, the right side) of the display moved with the central contour, so that the gap width between this texture field and contour remained constant. The width of the gap between con- 
tour and the texture field not tied to the motion of the contour (in Figure $3 b$, the texture on the left) changed as the two sides of the display approached and receded from one another. The width of the constant gap was varied to produce gaps of three sizes $(1,3.5$, and $5 \mathrm{~cm}$, respectively). For the small-gap, medium-gap, and largegap conditions, the width of the gap not linked to the contour by relative motion ranged from 0 to $2 \mathrm{~cm}$, from 2.5 to $4.5 \mathrm{~cm}$, and from 4 to $6 \mathrm{~cm}$, respectively. Thus, the average width of the varying gap was approximately equal to the width of the unvarying gap.

Display Condition 6. Displays in the relative-motion (subjectivecontour) condition also contained only relative-motion information (see Figure 3c). In the Figure 3c display, a static vertical subjective contour results from the presence of horizontal lines that begin on either side of the display and end at the same vertical midline. Lateral movements of the subjective contour were created in this condition by lengthening the horizontal lines on one side while the horizontal lines on the other side were simultaneously shortened by the same amount. As with the relative-motion (objectivecontour) condition, a small gap $(1 \mathrm{~cm})$ between texture and contour allowed for movement of the contour without the accretion/deletion of texture elements. Although the changing lengths of the horizontal lines themselves might be interpreted as accretion/deletion, the accretion (lengthening) of one set of lines was always balanced by an identical deletion (shortening) of the lines on the other side of the display. Thus, the accretion/deletion information available in these displays did not specify any depth ordering of surfaces (Gibson et al., 1969; Kaplan, 1969).

Display Conditions 7 and 8 . Two types of displays that lacked both relative-motion information and accretion/deletion information served as control conditions. In the first control condition, a single vertical line moved alternately left and right $(1 \mathrm{~cm}$ in each direction) across a dark homogeneous background. The second control condition was identical to the small-gap relative-motion (objective-contour) condition described above, except that there was no vertical line to serve as a contour. In this case, two laterally moving texture fields alternately approached and receded from each other. The amount of separation between the two texture fields in this condition ranged from 1 to $3 \mathrm{~cm}$.

\section{Procedure}

Before being tested, the subjects were given the following instructions: "Look at the display and decide which of the following is true: (1) the left side looks like it is in front; (2) the right side looks like it is in front; (3) both sides appear to be the same distance away."

The subjects were instructed to record their responses by pressing one of the three buttons, labeled "left," "equal," and "right," on the small box directly in front of them. Each subject received three practice trials in which two sheets of paper were held in front of the CRT by the experimenter and moved laterally so that they approached and receded from each other in a manner analogous to the computer-generated displays described above. The relative depth of the sheets of paper was varied so that the one on the left was closer to the subject on one trial, the one on the right was closer to the subject on another trial, and the sheets were equidistant from the subject on a third trial. The practice trials were presented in random order, and the subjects were asked to indicate which button represented the appropriate response. All subjects performed quickly and without error on the practice trials.

During test trials, the subjects observed displays monocularly from a distance of $3 \mathrm{ft}(.91 \mathrm{~m})$. The subjects viewed eight blocks of trials, corresponding to the eight conditions described above, with 20 trials in each block. The order of presentation for blocks was completely counterbalanced for the group as a whole. A new random distribution of texture elements was generated for each block of trials.

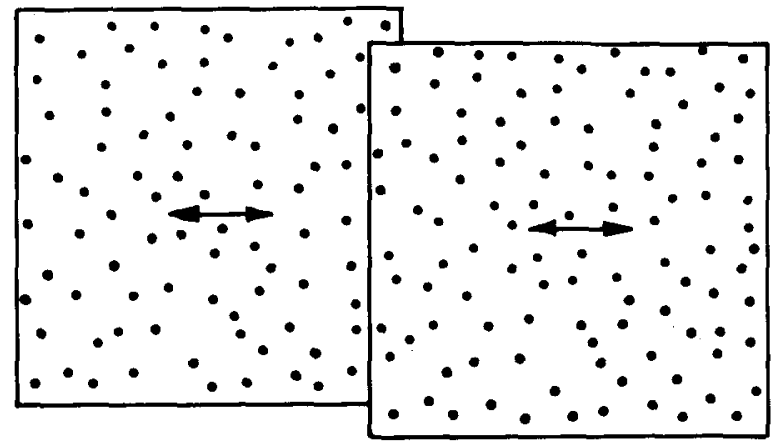

(a)

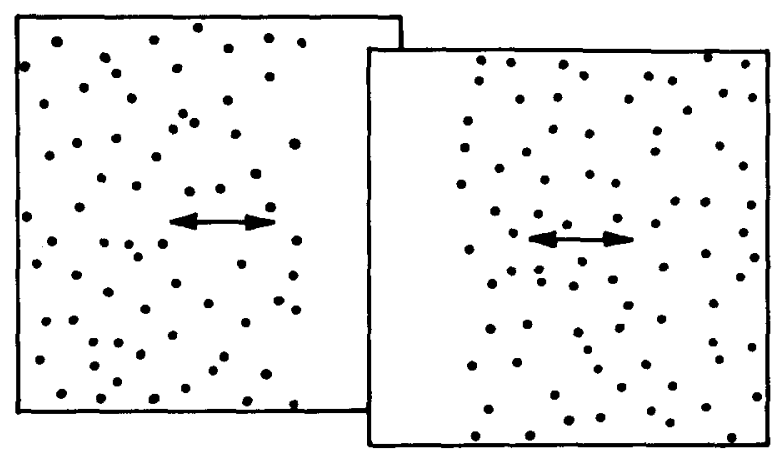

(b)

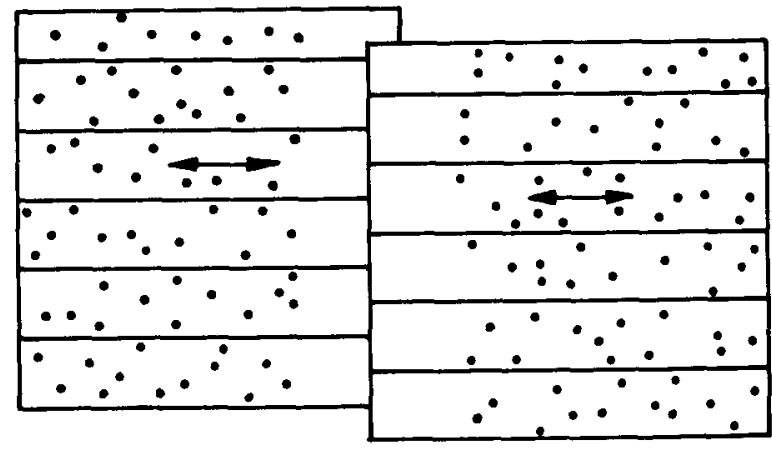

(c)

Figure 3. (a) Schematic drawing of accretion/deletion displays. In the subjective-contour condition, a subjective contour is perceived at the margin where accretion and deletion of texture occurs. In the objective-contour condition, a vertical line is located at the margin. (b) Relative-motion (subjective-contonr) display. "Gap" between vertical line and texture eliminates accretion/deletion information. (c) Relative-motion (subjective-contour) display. End-stopped horizontal lines generate a vertical subjective contour. Interposition displayed in these drawings was not present in computer-generated displays. 
Within a given block, the same random sequence of predicted depth orderings (right in front, left in front, neither in front) was presented to each subject. All displays continued without interruption until the subject recorded a response. After each response, there was a brief pause and then the next display was initiated. Total testing time was approximately $25 \mathrm{~min}$.

\section{RESULTS AND DISCUSSION}

The mean percentage of depth judgments consistent with predicted depth order and opposite predicted depth order and the mean percentage of "no depth" responses are presented in Table 1 . Two mixed-model repeated measures analyses of variance (ANOVAs) were conducted. To determine whether the experimental conditions yielded the perception of depth at an edge, a one-way ANOVA was carried out on the mean number of "no depth" responses for each of the eight conditions. This analysis revealed a significant main effect for condition $[F(7,105)$ $=28.07, p<.01]$. Post hoc comparisons based on Tukey's honestly significant difference method indicated that all six experimental conditions yielded significantly fewer responses of "no depth" than either control condition $(p<.05)$. To establish whether there were differences between the six experimental conditions in determining the perceived order of surfaces in depth, a second one-way ANOVA was carried out on the mean number of responses consistent with predicted depth order. This analysis yielded a significant main effect for condition $[F(5,75)=10.30, p<.05]$. Tukey post hoc comparisons revealed several significant differences $(p<.05)$, as shown in Table 2.

As the data in Table 1 show, the modified version of Kaplan's (1969) accretion/deletion (subjective-contour) displays used in this study were effective in producing the perception of depth at an edge. In addition, the reliable depth effect obtained in the accretion/deletion (objective-contour) condition indicates that visual processes sensitive to the depth information in these accretion/deletion displays are not disrupted when an ordinary vertical line serves as a contour.

Table 1

Mean Percentage of Depth Order Judgments as a Function of Display Condition

\begin{tabular}{|c|c|c|c|c|c|c|}
\hline \multirow[b]{2}{*}{ Condition } & \multicolumn{2}{|c|}{$\begin{array}{c}\text { Predicted } \\
\text { Depth Order }\end{array}$} & \multicolumn{2}{|c|}{ No Depth } & \multicolumn{2}{|c|}{$\begin{array}{l}\text { Opposite Predicted } \\
\text { Depth Order }\end{array}$} \\
\hline & Mean & $S D$ & Mean & $S D$ & Mean & $S D$ \\
\hline $\begin{array}{l}\text { Accretion/deletion } \\
\text { Subjective contour } \\
\text { Objective contour }\end{array}$ & $\begin{array}{l}98.8 \\
98.1\end{array}$ & $\begin{array}{l}2.8 \\
7.3\end{array}$ & $\begin{array}{r}0.6 \\
0\end{array}$ & 0.5 & $\begin{array}{l}0.6 \\
1.9\end{array}$ & $\begin{array}{l}1.6 \\
7.3\end{array}$ \\
\hline $\begin{array}{l}\text { Relative motion } \\
\text { Subjective contour }\end{array}$ & & & & & & \\
\hline $\begin{array}{c}\text { Small gap } \\
\text { Objective contour }\end{array}$ & 95.6 & 9.2 & 3.1 & 6.6 & 1.3 & 1.2 \\
\hline $\begin{array}{l}\text { Small gap } \\
\text { Medium gap } \\
\text { Large gap }\end{array}$ & $\begin{array}{l}87.2 \\
78.8 \\
62.5\end{array}$ & $\begin{array}{l}16.7 \\
19.7 \\
33.7\end{array}$ & $\begin{array}{l}10.0 \\
16.5 \\
24.4\end{array}$ & $\begin{array}{l}15.8 \\
16.7 \\
27.5\end{array}$ & $\begin{array}{r}2.8 \\
4.7 \\
13.1\end{array}$ & $\begin{array}{r}4.7 \\
7.0 \\
18.1\end{array}$ \\
\hline $\begin{array}{l}\text { Controls } \\
\text { Contour only } \\
\text { Texture only }\end{array}$ & & & $\begin{array}{l}67.5 \\
53.5\end{array}$ & $\begin{array}{l}30.7 \\
31.4\end{array}$ & & \\
\hline
\end{tabular}

The main purpose of the present study was to determine whether adults were sensitive to depth information specified by the relationship between the optical motion of a contour and the optical motion of texture elements on both sides of the contour. The depth effect obtained in each of the relative-motion conditions (see Table 1) indicates that when depth information from the accretion and deletion of texture is eliminated, adults perceive depth at an edge on the basis of relative-motion information alone. In addition, as the data in Table 2 show, the two relative-motion conditions with small gaps (the subjectivecontour condition and the objective-contour/small-gap condition) did not differ significantly from either of the two accretion/deletion conditions in determining the perceived order of depth.

It is possible, however, that subjects' judgments in the relative-motion conditions were based upon a response bias produced by the practice trials and/or the accretion/deletion displays. Prior exposure to these may have created a set, or response criterion, for interpreting the relative-motion displays. To rule out this possibility, a follow-up experiment was conducted in which 7 naive adult subjects viewed only a single continuous display of the small-gap relative-motion (objective-contour) condition. The procedure employed was similar to that used in the main study, except that the practice trials were eliminated and the subjects were simply asked to describe the display. Initially, no mention of depth was made. Three of the 7 subjects spontaneously reported seeing one surface moving over another, with the predicted depth order. When prompted with the question "Is there any depth suggested in the display?" the remaining 4 subjects all reported the predicted depth effect. This was the case even though no mention was made of what form the apparent depth might take. Thus, the depth effect obtained from relative-motion information in the absence of other cues seems quite robust; to our knowledge, sensitivity to this depth cue has not been previously demonstrated.

A second finding of the study may be informative about the mechanism used by the visual system in perceiving depth from relative-motion information. As the data in Table 2 show, both of the small-gap relative-motion (subjective-contour and objective-contour) conditions yielded significantly more responses consistent with predicted depth order than did the large-gap relativemotion (objective-contour) condition. In addition, as Table 1 indicates, the large-gap condition showed significantly more responses of "no depth" than did either of the accretion/deletion conditions. These results indicate that the depth effect obtained in the relative-motion conditions diminishes as the width of the gap between texture and contour is increased. One interpretation of this finding is that the detection of relative-motion information for depth depends on processes that are relatively local, namely, computations that compare the motion of texture elements that are relatively near the contour with the motion of the contour. However, it should be noted that even the large-gap relative-motion condition produced a perception of depth relative to control conditions (see Ta- 
Table 2

Comparison of the Experimental Treatment Effects

\begin{tabular}{|c|c|c|c|c|c|c|}
\hline \multirow[b]{3}{*}{ Condition } & \multirow{2}{*}{\multicolumn{2}{|c|}{ Accretion/Deletion }} & \multicolumn{4}{|c|}{ Relative Motion } \\
\hline & & & \multirow[b]{2}{*}{$\begin{array}{c}\text { Subjective } \\
\text { Contour } \\
\bar{X}^{*}=19.13\end{array}$} & \multicolumn{3}{|c|}{$\begin{array}{l}\text { Objective } \\
\text { Contour }\end{array}$} \\
\hline & $\begin{array}{c}\text { Subjective } \\
\text { Contour } \\
\bar{X}^{*}=19.75\end{array}$ & $\begin{array}{c}\text { Objective } \\
\text { Contour } \\
\bar{X}^{*}=19.63\end{array}$ & & $\begin{array}{c}\text { Small } \\
\text { Gap } \\
\bar{X}^{*}=17.44\end{array}$ & $\begin{array}{c}\text { Medium } \\
\text { Gap } \\
\bar{X}^{*}=15.75\end{array}$ & $\begin{array}{c}\text { Large } \\
\text { Gap } \\
\bar{X}^{*}=12.50\end{array}$ \\
\hline $\begin{array}{l}\text { Accretion/deletion } \\
\text { Subjective contour } \\
\text { Objective contour }\end{array}$ & & 0.12 & $\begin{array}{l}0.62 \\
0.5\end{array}$ & $\begin{array}{l}2.31 \\
2.19\end{array}$ & $\begin{array}{l}4.0 \dagger \\
3.88 \dagger\end{array}$ & $\begin{array}{l}7.25 \dagger \\
7.13 \dagger\end{array}$ \\
\hline $\begin{array}{l}\text { Relative motion } \\
\text { Subjective contour } \\
\text { Objective contour } \\
\text { Small gap } \\
\text { Medium gap } \\
\text { Large gap }\end{array}$ & & & & 1.69 & $\begin{array}{l}3.38 \\
1.69\end{array}$ & $\begin{array}{l}6.63 \dagger \\
4.94 \dagger \\
3.25\end{array}$ \\
\hline
\end{tabular}

Note-Values listed are differences in the mean number of responses consistent with predicted depth order. Each experimental condition was tested against the remaining five conditions by Tukey's honestly significant difference test. $\quad * p<.05$. †Maximum score $=20$.

ble 1), although the effect is quite weak. An alternative to the above interpretation is that as the width of the gap between texture and contour increases, the texture field and contour are less likely to be perceived as belonging to the same occluding surface. The finding that, in the relative-motion (objective-contour) conditions, subjects perceive surfaces ordered in depth implies that the visual system effectively "filled in" missing texture in the textureless gap to produce the perception of a continuous surface. This propagation of a surface from the border of the texture field to the contour is most likely to occur when the gap width is small. When the distance between texture and contour is quite large, however, the border of the texture field itself may generate a contour and thus prevent the propagation of an apparent surface.

The data in Table 2 also show that, in the small-gap relative-motion (objective-contour and subjective-contour) conditions, the two types of contours did not differ significantly in generating the depth effect. The mechanism that detects relative motion of contour and texture is able to utilize both sorts of contour information in producing the perception of depth.

\section{CONCLUSIONS}

A new source of kinetic information for depth at an edge was investigated using adult subjects. Whether a surface is occluded by, or is occluding, a second surface determines the relationship between the motion of optical texture projected by each surface and the motion of the contour projected by the edge between them. The relative motion of texture and contour is thus a potential cue for the order of depth at an edge. The results reported here indicate that adults are sensitive to this relative-motion information for the order of surfaces in depth. Earlier studies (Andersen \& Braunstein, 1983; Granrud et al., 1984; Kaplan, 1969) have been interpreted as providing evidence for human sensitivity to a different cue for depth at an edge, namely, the accretion and deletion of optical texture. Because these studies confound accretion/deletion information and relative-motion information, however, the present results suggest that the depth effects obtained may have been based on relative-motion information. Logically, the visual system may use relativemotion information alone, accretion/deletion information alone, or both in determining the order of surfaces in depth. Evidence that accretion/deletion may be used in the absence of relative-motion information has been obtained by Proffitt, Bertenthal, and Roberts (1984).

The Michotte screening effect (see Figure 1) may be explained more accurately by an account based on relativemotion information than one based on the accretion and deletion of texture elements. According to the relativemotion account, the inconsistent motion of the disk and contour specifies that the contour belongs to an occluding surface and that the disk is being occluded. In contrast, the accretion/deletion accounts as put forth by Gibson et al. (1969) and Kaplan (1969) emphasize the appearance and disappearance of texture elements. Since neither the disk nor the apparently occluding surface in Michotte's display are textured, the only element that could appear or disappear is the disk itself. It seems clear that it is the way in which the disk disappears that specifies its occlusion and not simply the fact that it disappears. For example, if the disk were to disappear by symmetrically contracting, it would appear to withdraw in depth.

The experimental results reported in this paper deal only with translational motion. Rotation in depth of objects with smooth surfaces produces a dynamic self-occlusion; in this case, the contour no longer corresponds to a fixed location on a surface but, rather, slides along it. As previously mentioned, dynamic self-occlusion results in a situation in which deletion and accretion regions are associated with the side of a boundary corresponding to the surface that is in front, not the surface that is behind. The relative visual motion of contours and surfaces, however, still in principle correctly indicates the occluding surface. (A mathematical analysis is presented in Thomp- 
son et al., 1985.) The basic principle is that although the surface and boundary do not have a common threedimensional motion, the projected visual motion is the same, near the boundary. Rotation in depth of smooth surfaces may thus provide another tool for separating the effects of accretion and deletion from those due to the relative motion of surfaces and boundaries. Unfortunately, an additional confounding effect is introduced. Rotation in depth produces optical flow values that vary significantly over object surfaces. For rotation in depth, the visual system may have difficulty in utilizing the relationship between the visual motion of a contour and the occluding surface generating it because that relationship may not hold except quite near the contour.

This study represents only an initial step toward an understanding of spatial perception based on relative-motion information. Much remains to be investigated. For example, a goal of future research will be to discover the precise nature of the relative-motion information that is used by the visual system in perceiving the order of surfaces in depth. Two independent sources of depth information existed in the relative-motion displays used in the present study: the common motion between contour and texture on the apparently occluding surface and the differential motion between contour and texture on the apparently occluded surface. The visual system may, in principle, use one or both of these types of relative-motion information in determining depth order for surfaces.

A second goal for future research will be to determine whether the visual mechanism that detects relative-motion information operates locally or globally. If the texture field in the large-gap relative-motion condition generated a contour at its border, this would prevent subjects from perceiving a single continuous surface extending from texture to contour. Thus, the lack of a strong effect in this condition may reflect disruption of the process that propagates a surface from texture to contour and does not necessarily imply a local process. By covarying the gap width between texture and contour and the density of texture elements, however, it may be possible to untie these two alternatives. When the density of texture elements is sufficiently low, the visual system may not generate a con- tour at the border of a texture field. If the process that detects relative motion between contour and texture is global, then we would expect a relative-motion (objectivecontour) display with large gap width and low texture density to produce a stronger depth effect than a display with large gap width and high texture density.

\section{REFERENCES}

Andersen, G. J., Braunstein, M. L. (1983). Dynamic occlusion in the perception of rotation in depth. Perception \& Psychophysics, 34, 356-362.

GIBson, J. J. (1950). The perception of the visual world. Boston: Houghton-Mifflin.

GIBson, J. J. (1966). The senses considered as perceptual systems. Boston: Houghton-Mifflin.

GrBson, J. J. (1979). The ecological approach to visual perception. Boston: Houghton-Mifflin.

Gibson, J. J., Kaplan, G., Reynolds, H., \& Wheeler, K. (1969). The change from visible to invisible: A study of optical transitions. Perception \& Psychophysics, 5, 113-116.

Granrud, C. E., Yonas, A., Smith, I. M., Arterberry; M. E., Gucksman, M. L., SoRKNes, A. C. (1984). Infants' sensitivity to accretion and deletion of texture as information for depth at an edge. Child Development, 55, 1630-1636.

KANIZSA, G. (1955). Margini quasi-percettivi in campi con stimolazione omogenea. Rivista di Psicologia, 49, 3-19.

KANIZSA, G. (1979). Organization in vision: Essays on Gestalt perception. New York: Praeger.

Kaplan, G. A. (1969). Kinetic disruption of optical texture: The perception of depth at an edge. Perception \& Psychophysics, 6, 193-198.

MARR, D. (1982). Vision. San Francisco: W. H. Freeman.

Michotte, A., ThInes, G., C Crabbe, G. (1964). Les complements amodaux des structures perceptives (Studia psychologia). Louvain: Publications Universitaires de Louvain.

Profftt, D. R., Bertenthal, B. I., Roberts, R. J. (1984). The role of occlusion in reducing multistability in moving point-light displays. Perception \& Psychophysics, 36, 315-323.

Schumann, F. (1904). Beiträge zur Analyse der Gesichtswahrnehmungen: 1. Einige Beobachtungen über die Zusammenfassung von Gesichtseindrücken zu Einheiten. Psychologische Studien, 1, 1-32.

Thompson, W. B., Mutch, K. M., Berzins, V. A. (1985). Dynamic occlusion analysis in optical flow fields. IEEE Transactions on Pattern Analysis and Machine Intelligence, PAM I-7, 374-383.

(Manuscript received June 16, 1986; revision accepted for publication October 8, 1986.) 